Conclusion The review provides an overview of research strategies and measures with application and purpose described. Producing a map of how asset-based approaches are evaluated and articulating key methodological differences helps researchers and practitioners select appropriate evaluation methods. There are a number of limitations, including the use of rapid review methods which may have missed other relevant evaluation approaches. Further methodological development is needed in this field and we welcome debate about ways to evaluate asset-based approaches.

\section{OP78 NEIGHBOURHOOD SOCIAL COHESION, ETHNICITY AND PHYSICAL ACTIVITY IN ADOLESCENTS: LONGITUDINAL EVIDENCE FROM THE ORIEL STUDY}

${ }^{1} \mathrm{~N}$ Berger*, 'DJ Lewis, ${ }^{2}$ EN Njagi, ${ }^{1} S$ Cummins. 'Social and Environmental Health Research, London School of Hygiene and Tropical Medicine, London, UK; ${ }^{2}$ Non-communicable Disease Epidemiology, London School of Hygiene and Tropical Medicine, London, UK

\subsection{6/jech-2017-SSMAbstracts.77}

Background Most adolescents do not achieve the recommended level of physical activity (PA) in the UK. Cultural norms - captured by ethnic identity - and social cohesion are aspects of the social environment that have the potential to influence health (behaviours). This study examines the relationships between social cohesion, ethnicity and three common types of PA in adolescents. The objectives are to test whether different types of PA have similar patterns of associations with social cohesion and ethnicity and to investigate confounding and interaction effects.

Methods We used longitudinal data from the Olympic Regeneration in East London (ORiEL) study. In 2012, 3088 adolescents aged 11-12 were recruited from 25 schools in four deprived and ethnically diverse boroughs of East London. Adolescents were followed-up in 2013 and 2014. Social cohesion was operationalised as trust in people living in the neighbourhood, measured on a four-point scale at wave 2. We grouped ethnic identities into eight categories. The outcomes were self-reported binary variables: walking to school, walking for leisure, and outdoor PA in the neighbourhood. We identified potential confounders. We fitted Generalised Estimated Equation models for each outcome, with a time trend and investigated potential interactions between the exposures and time. Final available case sample size was 4811 observations from 2157 adolescents.

Results We found evidence of associations between ethnicity and the PA outcomes. Adjustment for potential confounders (socioeconomic status, health and gender) did not attenuate the relationships. In the fully adjusted models, compared to White British, Black Africans had 1.49 higher odds of outdoor PA (95\% CI 1.06-2.07), Bangladeshis had 1.42 higher odds of walking to school (95\% CI 1.02-1.97) and all ethnic groups had lower odds of walking for leisure (estimated ORs varied between 0.37 and 0.71 ). While social cohesion was not associated with walking, there was evidence of a dose-response relationship with outdoor PA. A one-category increase in cohesion increasedthe odds of outdoor PA by 1.18 (95\% CI 1.071.30). We found no evidence of time*exposure interaction; exposures did not predict change in PA.

Conclusion This study contributes new findings to the evidence base on the social environment and PA. The ethnic diversity of the ORiEL study and its large sample size provided sufficient power to reveal how PA behaviours are patterned by ethnic groups. Further analyses will jointly model the three PA outcomes to better capture the dependency and associations between the exposures and the outcomes.

\section{OP79 INDIVIDUAL AND NEIGHBOURHOOD-LEVEL SOCIOECONOMIC FACTORS AND INCIDENCE OF TYPE 2 DIABETES IN OLDER AGE: RESULTS FROM A 14 YEAR FOLLOW-UP OF A COHORT OF OLDER BRITISH MEN}

${ }^{1}$ SE Ramsay, ${ }^{2} \mathrm{D}$ Roberts, ${ }^{3} \mathrm{AO}$ Papacosta, ${ }^{3} \mathrm{LT}$ Lennon, ${ }^{5} \mathrm{PH}$ Whincup, ${ }^{3} \mathrm{SG}$ Wannamethee* ${ }^{1}$ Institute of Health and Society, Newcastle University, Newcastle upon Tyne, UK; ${ }^{2}$ Whittington Health NHS Trust, Whittington Health NHS Trust, London, UK; ${ }^{3}$ Department of Primary Care and Population Health, UCL, London, UK; ${ }^{4}$ Population Health Research Institute, St George's, University of London, London, UK

\subsection{6/jech-2017-SSMAbstracts.78}

Background Current evidence linking socioeconomic factors to incident Type 2 Diabetes Mellitus (T2DM) in older populations is conflicting. We investigated the prospective association of individual socioeconomic position and neighbourhood-level socioeconomic deprivation with incident T2DM in older British men, and examined possible underlying factors.

Methods A socially-representative cohort of 3487 men, aged 60-79 years in 1998-2000, from 24 British towns was followed-up for 14 years for incident cases of T2DM. Individual socioeconomic position was based on social class derived from the longest-held occupation in middle-age, and was categorised into non-manual and manual groups. Neighbourhood-level socioeconomic deprivation was based on national Index of Multiple Deprivation (IMD) quintiles; a composite score of neighbourhood-level factors (income, employment, education, disability, crime, housing and living environment), with a higher score indicating greater deprivation. Follow-up on type 2 diabetes was obtained from reviews of general practitioner records and self-reported from questionnaires. Cox proportional hazards models were used to obtain hazard ratios (HR) and 95\% CI for incident diabetes according to social class and IMD quintiles. Prevalent cases of diabetes at baseline were excluded from the analyses.

Results During the follow-up of 14 years, there were 289 incident cases of T2DM (7.1 per 1000 person-years). Diabetes risk increased from higher to lower social class groups and from IMD quintile 1 (least deprived) to quintile 5 (most deprived) ( $\mathrm{P}$ for trend=0.001). Compared with non-manual social class groups, age-adjusted HR for manual groups was $1.58(95 \% \mathrm{CI} 1.24-2.01)$ - this was largely attenuated (1.38; 95\% CI 1.08-1.76) on adjustment for body mass index (BMI); adjustment for blood pressure, smoking, alcohol, physical activity, diet, medication and family history resulted in little attenuation while further adjustment for triglyceride levels attenuated the association. Compared with IMD quintile 1, the risk of incident T2DM was highest in IMD quintile 4 $(\mathrm{HR}=1.79$; 95\% CI 1.24-2.54). This largely attenuated on adjustment for BMI (HR=1.46; 95\% CI 1.02-2.10), and became non-significant after adjustment for lifestyle factors (smoking, alcohol, physical activity and diet).

Conclusion Manual social class and neighbourhood-level socioeconomic deprivation was associated with an increased risk of T2DM in older British men. For social class this was mostly explained by BMI and triglycerides. For neighbourhood-level socioeconomic deprivation it was largely explained by BMI and lifestyle factors. Our results support the need for public health initiatives specifically targeting obesity as a means 
towards reducing socioeconomic inequalities in type 2 diabetes in later life.

\section{OP80 ASSOCIATION OF OBJECTIVE AND PERCEIVED NEIGHBOURHOOD CHARACTERISTICS WITH POOR ORAL HEALTH IN OLDER AGE: RESULTS FROM A CROSS- SECTIONAL STUDY OF OLDER BRITISH MEN}

${ }^{1}$ SE Ramsay*, ${ }^{2} \mathrm{E}$ Papachristou, ${ }^{2} \mathrm{AO}$ Papacosta, ${ }^{2} \mathrm{LT}$ Lennon, ${ }^{3} \mathrm{PH}$ Whincup, ${ }^{2} S G$ Wannamethee. ${ }^{1}$ Institute of Health and Society, Newcastle University, Newcastle upon Tyne, UK; ${ }^{2}$ Department of Primary Care and Population Health, UCL, London, UK; ${ }^{3}$ Population Health Research Institute, St George's, University of London, London, UK

10.1136/jech-2017-SSMAbstracts.79

Background Socioeconomic inequalities in oral health are established. However, the influence of neighbourhood-level socioeconomic factors on the oral health of older people is not well-established. We investigated both objective and perceived neighbourhood characteristics and their associations with a range of oral health measures in older age.

Methods The British Regional Heart Study comprises a representative sample of men drawn from 24 general practices across Britain at 40-59 years in 1978-80. In 2010-12, the participants when aged 71-92 years had a follow-up a physical examination including a dental assessment $(n=1622)$, and completed a questionnaire $(n=2137)$. Oral health assessment included objective measures (tooth count and periodontal (gum) disease), and self-reported fair/poor oral health and dry mouth symptoms. Neighbourhood deprivation was based on the Index of Multiple Deprivation (IMD); a composite score of neighbourhood-level factors (income, employment, education, disability, crime, housing, living environment). Perceived neighbourhood characteristics included local area services, safety, environment, and a cumulative index of these characteristics. Multilevel and multivariate logistic regression models were used to obtain odds ratios according to quintiles of IMD and perceived neighbourhood characteristics.

Results The risk of periodontal disease and tooth loss increased from IMD quintile 1 (least deprived) to 5 (most deprived); age-adjusted odds ratios (OR) for quintile 5 were 3.25 (95\% CI 2.05-5.17) and 3.58 (95\% CI $2.38-5.39)$ respectively, compared to quintile 1 . These associations were attenuated only slightly on adjustment for individual social class, smoking, depression, social interactions and history of cardiovascular disease or diabetes, and remained statistically significant. Age-adjusted odds of dry mouth was increased only in quintile $2(\mathrm{OR}=1.41,95 \% \mathrm{CI} 1.04-1.91)$ and quintile 5 (1.50, 95\% CI 1.09-2.07) compared to quintile 1 and was not significant after adjustments for the remaining covariates. The odds of self-reported fair/poor oral health was greater only in quintile $5(\mathrm{OR}=1.73$, 95\% CI 1.28-2.35), and remained statistically significant after adjustment for covariates. For perceived neighbourhood characteristics, significant trends were observed across quintiles of local area services, safety and a cumulative index of neighbourhood characteristics, with greater levels of tooth loss, periodontal disease, fair/poor selfrated oral health and dry mouth from quintile 1 (best rated) to quintile 5 (worse rated).

Conclusion Markers of poor oral health in older age were associated with both objective and perceived neighbourhoodlevel socioeconomic factors. Wider socioeconomic determinants are potentially important influences on the oral health of older people. Prospective studies are needed to establish these associations.

\section{Friday 8 September 2017}

\section{Physical activity interventions}

\section{OP81 LONG-TERM OBJECTIVE PHYSICAL ACTIVITY DATA FROM TWO PRIMARY CARE PEDOMETER-BASED RANDOMISED CONTROLLED TRIALS IN MIDDLE-AGED AND OLDER ADULTS- ARE THERE STILL POSITIVE TRIAL EFFECTS AT 3 AND 4 YEARS?}

${ }^{1} \mathrm{E}$ Limb*, ${ }^{1} \mathrm{~T}$ Harris, ${ }^{2} \mathrm{~S}$ Kerry, ${ }^{3} \mathrm{C}$ Victor, ${ }^{4} \mathrm{~S}$ Iliffe, ${ }^{1} \mathrm{M}$ Ussher, ${ }^{1} \mathrm{P}$ Whincup, ${ }^{1} \mathrm{C}$ Furness, ${ }^{1} \mathrm{C}$ Wahlich, ${ }^{1} \mathrm{D}$ Cook, ${ }^{5} \mathrm{U}$ Ekelund, ${ }^{6} \mathrm{~J}$ Fox-Rushby, ${ }^{1} \mathrm{~J}$ Ibison, ${ }^{1} \mathrm{~S}$ DeWilde. ${ }^{1}$ Population Health Research Institute, St George's University of London, London, UK; ${ }^{2}$ Pragmatic Clinical Trials Unit, Queen Mary's University of London, London, UK; ${ }^{3}$ Gerontology and Health Services Research Unit, Brunel, University of London, London, UK; ${ }^{4}$ Research Department of Primary Care and Population Health, University College of London, London, UK; ${ }^{5}$ Department of Sports Science, Oslo University, Norway; ${ }^{6}$ Health Economics Research group, Brunel, University of London, UK

\subsection{6/jech-2017-SSMAbstracts.80}

Background The PACE-UP 3-armed primary care trial recruited 45-75 year olds into a 12 week pedometer-based intervention, with one postal intervention arm and one nurse support. The PACE-Lift 2-armed primary care trial recruited 60-75 year olds into a 12 week nurse-supported pedometerbased intervention. Both trials increased step-counts by around a tenth and time in moderate-to-vigorous physical activity (MVPA) in bouts by around a third at 12 months, with no difference between nurse and postal arms in PACE-UP. Longterm physical activity (PA) maintenance, particularly MVPA in bouts, is important for a wide range of health benefits, but few trials provide objective PA measures beyond 12 months. We followed up PACE-UP and PACE-Lift cohorts at 3 and 4 years respectively, to investigate whether intervention effects persisted.

Methods 3 year (PACE-UP) and 4 year (PACE-Lift) accelerometer outcomes were regressed on baseline accelerometry to estimate change in average daily step-counts and average weekly time in MVPA in $\geq 10 \mathrm{~min}$ bouts in the treatment groups compared to control groups. Imputation analyses were conducted to account for missing data effects.

Results PACE-UP 3 year follow-up rate was 67\% (681/1023). Both intervention groups were still doing more steps/day than the control group: postal 627 (95\% CI 198, 1056); nurse 670 (95\% CI 237, 1102); nurse plus postal 648 (95\% CI 272, 1024). The pattern was similar for total weekly MVPA in bouts: postal 28 (95\% CI 7, 49); nurse 24 (95\% CI 24 (3, 45); nurse plus postal 26 (95\% CI 8, 44). PACE-Lift 4 year follow-up rate was 76\% (225/298). In PACE-Lift the intervention versus control comparisons were 407 (95\% CI -177, 992) for steps and 32 (95\% CI 5, 60) for MVPA in bouts; though the steps comparison is not statistically significant, both the steps and MVPA estimates are consistent with the PACE-UP findings. Both trials showed no wear-time differences between groups and imputation analyses did not change results interpretation.

Conclusion Over two thirds of both trial cohorts provided long-term data. Both trials showed persistent effects on time in MVPA in bouts at 3 or 4 years. PACE-UP showed a 